The posterior tibial and dorsal artery of the foot could be felt pulsating.

Next day, at 3 P.M., Mr. Macnamara applied an Esmarch's elastic bandage from the foot to the lower border of the aneurism, and a second round the thigh above the knee, leaving the tumour uncovered. Owing to the patient being unable to bear the pain, ether was administered at 3.17 and continued till 3.55. Digital compression was then applied to the femoral artery, and the Esmarch bandage was removed. No pulsation was felt in the aneurism. At 5 P.M. the leg was flexed on the thigh, and, having been fixed in that position, compression of the femoral artery was discontinued. Slight pulsation was now felt in the aneurism.

On the 6th pulsation was more marked in the aneurism than on the previous evening.

On the 10th Mr. Macnamara, at 1.30, again applied Esmarch's bandage, carrying it up to the lower border of the aneurism, then a web elastic bandage was put on lightly over the knee, including the popliteal space and aneurism, and carried up to the lower one-third of the thigh. Ten minutes after the application of the bandage chloroform was administered, and the patient kept under its influence till 2.30. Digital compression having been applied to the femoral artery, the Esmarch's bandage was removed. On relaxing the compression a slight pulsation could be felt in the aneurism. Digital compression was kept up until 5 P.M. On being discontinued no pulsation was felt in the tumour. The leg was semi-flexed on the thigh, and the parient was kept in bed until the 19th of July.

No pulsation returned in the aneurism, a marked decrease in the size of the tumour could be felt every day, and the patient was discharged from the hospital on the 28th of the month.

He was seen six months after leaving the hospital. No perceptible remains of the aneurism could be detected, and the patient had long since returned to his work.

\section{VICTORIA DOCK DISTRICT DISPENSARY.}

SEVEN CONSECUTIVE CASES OF PLEURISY WITH EFFUSION; PARACENTESIS ; REMARES.

(Under the care of Mr. JULuUS CASAR.)

CASE 1.-Thos. M-, aged fourteen years, presented himself, complaining of a short, dry cough and shortness of breath. He had been under treatment elsewhere for two months for the same symptoms. On examination the left side of the chest was seen to be much larger than the right and measured one inch and a half more; dulness reached as high as the spine of the scapula behind, and to the third interspace in front. Respiratory murmur, vocal resonance, and vocal fremitus were absent. The heart's apex was pushed over to just inside the right nipple, all the signs of pleuritic effusion being well marked. The aspirator was used and thirty ounces of serum drawn off, when the patient complained of faintness, and the operation was stopped. The faintness soon passed off, and in a week he was able to resume his employment, that of an errand boy.

CASE 2. - A girl, aged seven years, complained of a pain in the right side, short, dry cough, shortness of breath, and feverishness. On examination friction sounds were heard over a small area at the angle of the scapula. A diuretic mixture was ordered and blistering fluid was painted over the side, followed by poultices. In four days more effusion was diagnosed. The same treatment was continued for a week, at the end of which time, the amount of effusion having undergone no diminution, tapping was resorted to, and eight ounces of serum were evacuated. In a week's time she was able to resume her attendance at school.

CASE 3.-A boy, aged three years, complained of pain in the right side, short cough, inability to lie on the affected side, shortness of breath, and feverishness. Examination revealed a small patch of dulness at the angle of the scapula, with diminished respiratory sounds, vocal resonance, and vocal fremitus. Two ounces of serum were drawn off, and in three days he was able to resume attendance at school.

CASE 4. - Male, aged three years, presented himself with a history similar in every respect to Case 3. A diuretic mixture, counter-irritants, and poultices were ordered and continued for ten days, but producing no beneficial effect, aspiration was had recourse to, and five ounces of serum were drawn off. Iodine liniment and a stout flannel roller were applied to the side. This treatment relieved all sym. ptoms very much, but only for a few days, when his breathing became very much embarrassed. The temperature went up to $103^{\circ}$, and he began to have night sweats. Dul. noss now reached to within three fingers' breadth of the spine of the scapula behind, and to within two of the clavicle in front. All the symptoms pointing to empyema, tapping was determined upon, and fifteen ounces of thick pus evacuated. The iodine liniment and bandage treatment were resumed. The patient made a rapid and complete recovery.

CASE 5.-A tall and very anæmic girl, aged fifteen years, complained of all the usual symptoms, and presented most of the usual signs of pleuritic effusion. As her mother was at first unwilling to consent to tapping, tonics with iodide of potassium were administered internally, and counter. irritants and poultices were applied externally for a fortnight, but without benefit. The mother now consenting, the aspi. rator was used, and the fluid withdrawn. After this she improved rapidly, and was able to resume her occupation (that of a barmaid) at the end of a fortnight.

CASE 6 was that of a strong, healthy woman, aged twenty. six years, who, on presenting herself for treatment, complained of a stabbing pain just outside the right nipple, slight shortness of breath, short cough, and inability to lie on the affected side. Examination revealed friction sounds at the angle of the scapula, where there was slight dulness, with diminution of respiration sounds, vocal resonance, and voeal fremitus. Diuretic mixture and courter-irritants were ordered at the end of a week. Not having made any im. provement she was ordered to wear a stout flannel roller and to continue the other treatment for a week, at the end of which time, the amount of effusion not having diminished, tapping was resorted to, and twenty-nine ounces of serum withdrawn, after which she made a quick recovery.

CASE 7.-Male, aged twenty-one years. This case was in every particular similar to Case 6 , except that a fortnight after the first tapping a second effusion into the same sidethe right-took place, necessitating a second use of the aspirator. He was able to resume work in a week's time.

Remarles. - I think these cases are of interest, as showing, as far as things go-lst. The superionity of tapping, at least as regards time, over drugs. 2nd. That early taping will effect an early cure. 3rd. That cogophony is rather a curiosity, in effusions from simple pleuritis, than otherwise. 4. That all cases of empyema do not require the use of in jections for their cure.

\section{LIVERPOOL NORTHERN HOSPITAL.}

\section{VESICAL CALCULUS ; LATERAL LITHOTONY ; SUBSEQUENT} DISCHARGE OF PUS FROM UMBILICUS; RECOVERY.

(Under the care of Mr. Cratuncy PUZex.)

ThE following case seems exactly similar to one reported by Mr. Bryant, of Guy's Hospital, in THE LANCET for Feb. 26th of this year.

Thomas $\mathrm{H}-$, a delicate, rickety-looking boy, ten years of age, was admitted on Jan. 28th, 1880. He was suffering from frequent painful micturition, the urine being loaded with phosphatic deposits and mucus, and having an alkaline reaction. On sounding, a large, rough calculus was at once detected.

On March 3rd, lateral lithotomy was performed in the ordinary manner, but on attempting the withdrawal of the stone it was found that in conseguence of its size, and the small development of the child, extraction could hardly be effected without risk either of unduly bruising the neck of the bladder or of crushing the stone in the forceps. The right side of the prostate was therefore slighily incised with a hernia-knife, and then the calculus was readily extrected. It weighed within a few grains of an ounce, bnt was large compared with its weight, the main body of the calculus being composed of uric acid thickly coated with loosely arranged layers of phosphatic deposits. The boy suffered no pain or discomfort from the operation, but the wound was a long time in closing; the urine was generally turbid and alkaline, and the surface of the wound often corered with muco-pus and with phosphatic deposit. This state of affairs was to a certain extent successfully combated by daily washing out the bladder (by means of a catheter passed through the wound) with a weak solution of nitric acid. His general condition was decidedly improring. He had been in a miserable state on admission, aud it appeared 